\title{
TECHNOLOGICAL INNOVATION AND COMPLEXITY THEORY
}

\author{
KOEN FRENKEN* \\ Urban and Regional Research Centre Utrecht (URU), Faculty of Geosciences, Utrecht University, \\ Heidelberglaan 2, P.O. Box 80115, 3508 TC Utrecht, The Netherlands
}

(Received 4 January 2005; Revised 2 March 2005; In final form 15 April 2005)

\begin{abstract}
Complexity theory has become influential in recent models in social science. In the context of innovations and new technologies, most applications have focused on technology adoption and technology diffusion, whereas the topic of the innovation process has received less attention. This paper discusses three families of complexity models of technological innovation: fitness landscape models, network models and percolation models. The models are capable of analysing complex interaction structures (between components of technologies, between agents engaged in collective invention) while avoiding 'over-parameterisation'. The paper ends with discussing the methodological challenges and critiques regarding the application of complexity theory that remain.
\end{abstract}

Keywords: Innovation; Complexity; Fitness landscape; NK-model; Percolation; Complex networks

JEL Classification: O3, B52

\section{INTRODUCTION}

Formal modelling has always been an important part of research on technological innovation. In particular, evolutionary economists developed a family of models addressing technology adoption, diffusion and increasing returns (Fisher and Pry, 1971; Metcalfe, 1988; Arthur, 1989; David, 1993; Bruckner et al., 1996; Dalle, 1997; Bikchandani et al., 1998) and the wider role of technical change in industrial dynamics and economic growth (David, 1975; Nelson and Winter, 1982; Winter, 1984; Silverberg et al., 1988; Silverberg and Verspagen, 1994; Klepper, 1996; Malerba et al., 1999; Fagiolo and Dosi, 2003). Though these models have been very important in establishing an evolutionary understanding of technical change, their contribution in terms of understanding the innovation process itself has been less important. Models deal either with technology adoption given a set of technologies or with innovation as a simple stochastic process. This research strategy is perfectly legitimate if the main interest is to understand the role of technical change in the economy.

More recently, a new cohort of models focuses on technological innovation as a complex phenomenon making use of new modelling techniques from complexity sciences. The use of complexity theory in economics is an emerging field of research (Anderson et al., 1988; Leydesdorff and van den Besselaar, 1994; Arthur et al., 1997; Foster and Hölzl, 2004). The

\footnotetext{
*E-mail: k.frenken@geo.uu.nl
} 
important contribution of complexity theory is its ability to model more complex interaction structures with less parameters. In this way, realism can be added to previous models without sacrificing analytical rigour. Regarding the topic of technological innovation, two frames of reference are relevant: (i) complexity can refer to complex interaction structures of components in a technological system and (ii) complexity can refer to structures of interactions between agents in innovation networks. Complexity theory proves to be applicable in both domains.

Here, we will not provide a review of complexity models in the rich field of the economics of technical change. Reviews on subsets of complexity models have been numerous recently, including Cowan (2004) on network models of innovation, Dawid (2004) and Pyka and Fagiolo (2005) on agent-based models of innovation, Llerena and Lorentz (2004) on technical change and evolutionary macroeconomics, Silverberg (2003) on long waves in technical change, and Windrum (2004) on neo-Schumpeterian models of industrial dynamics. Rather, the goal of this paper is to critically discuss complexity models of technological innovation (thus excluding related topics of technology adoption, diffusion, industrial dynamics and economic growth). To this end, we will first provide a definition of complexity that is useful in the context of technological innovation in order to delineate our discussion from the wider and rapidly expanding literature on complexity (Sec. 2). Then, we distinguish between three research lines on technological innovation that make use of complexity theory being fitness landscape models, complex network models and percolation models (Sec. 3). We end with a reflection on challenging methodological issues, which have remained, and promising research avenues ahead (Sec. 4).

\section{DEFINITION AND DELINEATION}

Complexity theory encompasses a wide range of theoretical contributions in most scientific disciplines. Among the many definitions that have been proposed, a common one that we deploy here, is that complexity theory deals with the study of complex systems that are composed of many interacting elements that interact in complex ways (Simon 1969). Following this definition, the structure of interactions between elements is of main interest. Complex systems can be described as a graph with nodes (elements) and edges (interactions). The structure of interactions is then given by the topology of a graph. One way to define complexity is by the number of interactions that exist between elements. The maximum complexity of a system can then be expressed as a function of the number of elements $N$. Assuming that interactions are not necessarily mutual (a directed graph), the maximum number of interactions is given by $N(N-1)$.

One can define complex systems more specifically as the class of graphs with a nontrivial topology, that is, a topology that is neither completely empty (no elements interacts with any other element) nor completely full (all elements interact with all other elements). Empty graphs cannot really be considered systems because the elements have no relations with other elements. Fully connected graphs, though seemingly 'complex', turn out to be easy to understand mathematically as all elements have the same position vis-à-vis all other elements.

Another way to further define complex systems is to include the possibility of strong and weak interactions. Instead of defining complex systems as consisting of elements with dichotomous interactions (present or absent), one can describe interactions between elements along a continuum. In the latter case, one can define complex systems as systems in which all elements interact with all other elements, thus fully connected, yet in which the interactions vary in strings (e.g. as expressed as a value between 0 and 1).

The preferred definition of complexity is dependent on the specific model and phenomenon that the model intends to describe. However, what unites much of the complexity research, 
including the three applications to the topic of technological innovation discussed subsequently, is that the degree of complexity of systems can be tuned with a single parameter. Thus, although complexity models focus on the complex nature of interdependencies between elements, 'over-parameterisation' can be avoided. This is of great importance because analytical solutions are more often than not impossible to obtain from complexity models due to feedback loops and stochasticity. Instead, the model's behaviour is usually analysed by simulation, which is feasible with a limited number of parameters. Complexity modelling should thus not be mistaken for complicated models with many parameters and possible behaviours (Axelrod, 1997). ${ }^{1}$

The interdisciplinary nature of complexity theory can be readily understood from the fact that complex systems exist in natural worlds (fluids, ecosystems and weather), social worlds (organisations, markets and societies) and artificial worlds (technologies, institutions and languages). Considering the topic of technological innovation, two dominant frames of reference are of relevance. First, there are models in which systems refer to technological systems containing many components that interact in complex ways. For example, an aircraft consists of many different components and subsystems that interact physically in complex ways. This means that an innovation in one part of a technological system almost as a rule causes problems elsewhere in the system. Innovation, in this view, is best viewed as a complex combinatorial optimisation problem. Second, there are models in which systems refer to populations of agents that interact in complex ways. This class of models deals with complex interaction structures between agents that collectively contribute to innovation (via knowledge spillovers, trade, collaboration). The precise structure of relationships in the network affects the performance of a network, for example, in terms of the speed of innovation and diffusion. Both models of complex technologies and models of interacting agents are discussed later. Fitness landscape models (Sec. 3.1) are applicable to the evolution of complex technological systems, whereas complex network models (Sec. 3.2) and percolation models (Sec. 3.3) are applicable to both technological systems and population of agents.

A final distinction that is useful in our discussion of complexity models of technological innovation is between exogenous complexity models and endogenous complexity models. Exogenous complexity models are characterised by a topology of interactions between system elements that is parameterised ex ante and remain unchanged over time (during the simulation). The behaviours of the model can be analysed by tuning, as a modeller, the complexity parameter from lowest values to highest value. In endogenous complexity models, in contrast, the topology of interactions evolves itself over time, which means that the degree of complexity is no longer controlled by the modeller using a parameter. We will see that in all three families of complexity models (fitness landscapes, networks and percolation), there are examples of exogenous and endogenous complexity models of innovation, although it is fair to say that, hitherto, most emphasis has been on exogenous complexity.

\section{COMPLEXITY MODELS OF INNOVATION}

\subsection{Fitness landscapes}

Understanding technologies as complex systems means that one has to define its elements and their interactions. Let $N$ be the number of elements in a system and $i$ an index for each element

\footnotetext{
${ }^{1}$ Some models of technological innovation in evolutionary economics suffer from over-parameterisation, which means that the possible behaviours of the models for different parameters were not always properly understood. This problem has become less urgent given the rapid increases in computing power; yet, even some recent contributions do not systematically explore the whole parameter space (on this, see Windrum, 2004).
} 
$(i=1, \ldots, N)$. For example, a car can be described by a number of elements including the engine, brakes, transmission, steering device, etc. To design a technological system, there is generally more than one option for each element. Assuming (without loss of generality) that there are two options for each element, the total number of possible designs adds up to $2^{N}$. Thus, even for systems with a small number of elements, the total number of possible designs is large. The space (hypercube) of possible designs is called the design space of a technology. Technological innovation can then be understood as a move of an agent (the designer) from its current location in the design space to a new location. Formally, this representation is equivalent to mutation in biological organisms, which involves a move from one string (say 00110) to another string (say 10110) in genotype space.

Technological innovation, generally, does not only involve a move in design space, but also an improvement in performance. Thus, when a designer is searching the design space of a technology, it is looking for strings with high performance or 'fitness'. Trial-and-error search can then be defined as an algorithm that randomly changes one or more elements (from 0 to 1 or vice versa), evaluates whether the fitness of the newly found string exceeds the fitness of the old string and moves to the new string if a fitness increase can indeed be realised. If one assumes that a designer searches myopically (Atkinson and Stiglitz, 1969), it means that innovation involves the mutation of only one element at the time. Put differently, in myopic search a designer searches only in the direct neighbourhood of the present design, where neighbourhood stands for the subset of strings that can be reached by mutation in one element. Trial-and-error search will halt once a designer has found a string with fitness that exceeds the value of all its neighbouring strings. Using the metaphor of a fitness landscape that maps the fitness values onto the strings in the design space, myopic search halts at a peak in the landscape, which may well be only locally optimal and not globally optimal, which would require a string to have the highest fitness of all strings in the design space. ${ }^{2}$

\subsubsection{The NK-model}

The landscape of fitness values, defining local and global optima, is dependent on the interactions between elements what is called 'epistatic relations'. The set of epistatic relations between elements make up a certain structure, which we call here a technology's architecture. Architectures range in complexity from fully decomposable architectures (no epistasis) to fully epistatic architectures (each element affects all other elements). Kauffman (1993) restricted his analysis to particular types of architectures, the complexity of which can be expressed in a single parameter. In his model, he used only those architectures in which each element is epistatically affected by the same number of other elements. The number of elements by which each element is affected, is indicated by parameter $K$. These so-called 'NK-systems' are then defined as systems with $N$ elements in which each element is affected by $K$ other elements. ${ }^{3}$

Kauffman's (1993) NK-model simulates the effects of epistasis by constructing a fitness landscape, a concept introduced by Wright (1932) in biological theory. In the context of technology, fitness landscapes can be taken to refer to efficiency (for production processes) or quality (for products). In a fitness landscape, each string in design space is assigned a fitness

\footnotetext{
${ }^{2}$ Other approaches to fitness landscapes assuming a continuous design space are discussed by Hartmann-Sonntag et al. (2004). See also Kwasnicki (1996).

${ }^{3}$ For application of the NK-model to technological innovation, see Frenken et al. (1999), Auerswald et al. (2000), Kauffman et al. (2000), McCarthy (2003, 2004), Frenken and Nuvolari (2004), Hovhannisian (2004), Hovhannisian and Valente (2004), Sommer and Loch (2004) and Frenken (2005). For applications of the NK-model in organisation theory, see Kauffman and Macready (1995), Levinthal (1997), Levinthal and Warglien (1999), Gavetti and Levinthal (2000), Marengo et al. (2000), Rivkin (2000, 2001), Dosi et al. (2003) and Rivkin and Siggelkow (2003).
} 

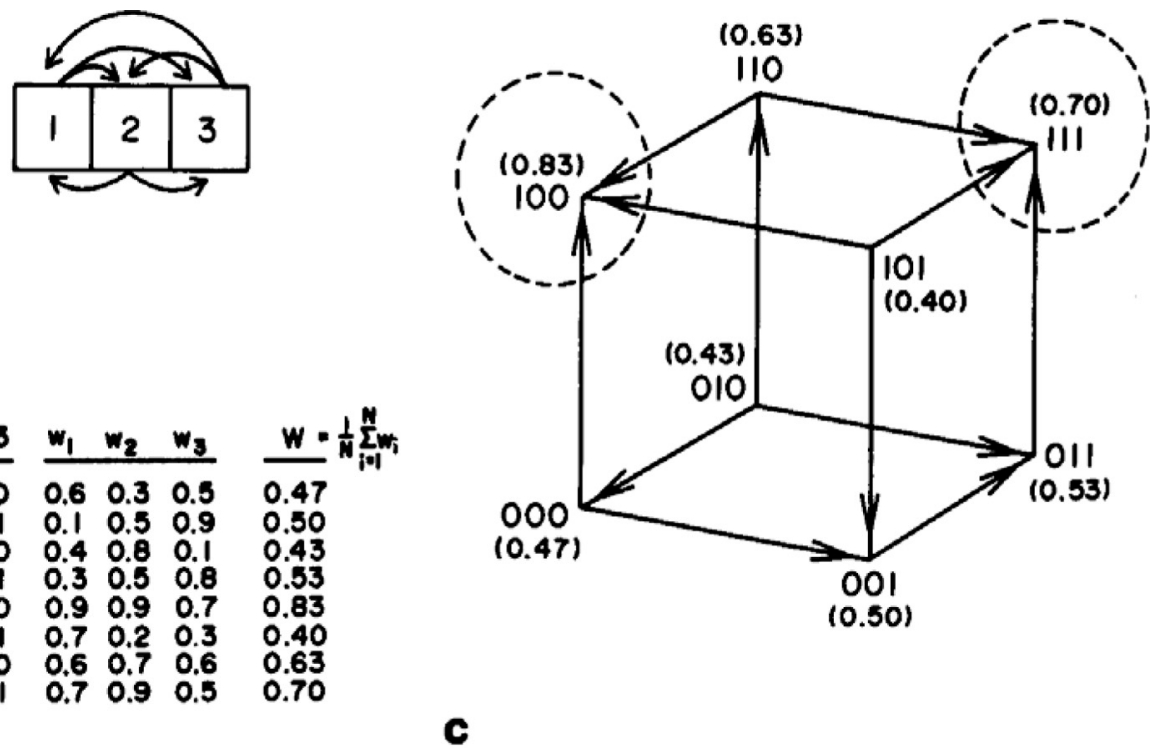

FIGURE 1 (a) Architecture of epistatic relations ( $N=3$ and $K=2$ ), (b) fitness table and, (c) fitness landscape (from Kauffman, 1993: 42).

value. In the NK-model, the fitness value $W$ of a string $s$ is computed as the mean of the fitness values $w_{i}$ of each element $i$ :

$$
W(s)=\frac{1}{N} \cdot \sum_{i=1}^{N} w_{i}(s)
$$

Due to epistasis a fitness value $w_{i}$ takes on a different value when element $i$ is mutated or when another element mutates that epistatically affects element $i$. Each time such a mutation takes place the fitness value of this element is redrawn randomly from a uniform distribution $[0,1]$. For $K=0$, epistasis is absent and $w_{i}$ takes on only two random values dependent on state 0 or 1. For $K=N-1$, in contrast, all elements affect each other, which means that any mutation in an element will produce new fitness values for each element in a string. In that case, the fitness value of an element $w_{i}$ has to be determined for each different string in design space. Thus, one has to draw $N$ times $2^{N}$ a random value. The latter case of maximum complexity $(K=N-1)$ is simulated for a system with three elements in Figure 1. As explained, for a string to be a peak in the landscape its $N$ neighbouring strings should have a lower fitness value. In the simulation in Figure 1, there are two strings that correspond to peaks ( 100 and $111)$.

Two important properties of NK fitness landscapes are relevant in the context of technological innovation (Kauffman, 1993): ${ }^{4}$

1. The number of local optima increases with increasing $K$ and with increasing $N$. This means that for systems with some $N$ and higher complexity $K$, and for systems with some $K$ and higher $N$, it becomes increasingly more likely that myopic search leads to a local optimum rather than the global optimum.

2. The mean fitness of local optima is highest for systems with a positive but moderate complexity (around $K=2, K=3$ or $K=4$ depending on $N$ ). This can be understood as

\footnotetext{
${ }^{4}$ For formal properties of NK landscapes, see Kauffman (1993) and the review chapter by Altenberg (1997).
} 
reflecting that small $K$-values create, on average, positive complementarities. When $K$ values get larger, they pose increasingly more incompatible design constraints resulting in poorer performance of local optima. For $K$ and $N$ are infinitely large, the expected fitness tends towards the expected value of a random draw ${ }^{5}$, which equals 0.5. Kauffman (1993: 54) called this result the 'complexity catastrophe'.

Result (1) means that the more complex a technology, the less effective local search will be. This explains, at least in some general sense, that complex technologies (large $K$ and $N$ ) are typically developed by professional R\&D labs with a science base that goes beyond local search, whereas relatively simple technologies (small $K$ and/or small $N$ ) can, in principle, be developed by individual inventors. Result (2) implies that systems of moderate complexity produce, on average, the highest fitness values of local optima. Consequently, the expected performance of a technology is highest when designers deal with moderately complex systems, and not, as one would perhaps expect, when dealing with systems without any epistasis $(K=0)$. The reason is that systems without epistasis leave no room for possible complementarities between individual elements.

Many search strategies other than myopic search are possible, and the conclusions for myopic search above do not necessarily carry over to models with different search algorithms. One alternative algorithm, which has been analysed by Frenken et al. (1999) and Kauffman et al. (2000), is a search strategy that allows multiple elements to be changed simultaneously. In this context, search distance is defined as the number of elements an agent can change simultaneously. Frenken et al. (1999) showed that the search distance that is required to find the global optimum with full certainty is dependent on the decomposability of a system. ${ }^{6}$ More precisely, the search distance should equal the size of the largest of the subsystems in which a system can be decomposed (called the cover size of a system, see Page 1996). In addition, systems that are nearly-decomposable in that most epistatic relations are contained in subsystems and only few epistatic relations exist between subsystems, can also be searched effectively using a search distance that corresponds to the size of the largest subsystem (Frenken et al. 1999; Simon, 2002). Note that search strategies with varying degrees of search distance constitute only one out of many classes of search strategies that can be analysed systematically on their performance using fitness landscape models. ${ }^{7}$

An important topic that can be explored with fitness landscapes is imitation. From the literature on technological innovation, it is known that some fraction of firms relies on other firms in that they imitate the innovative solutions found by other firms. Imitation can be defined as an agent copying the exact string of another agent exploring the landscape. However, imitation is failure-prone as it is generally difficult to copy the exact technology of another firm, including the competencies and organisational structures that complement the technology of a firm (Teece et al., 1997). As Rivkin (2000) showed, any error in copying the states of elements of a string can result in a drastic fall in fitness due to epistatic relations between elements. The fall in fitness due to inexact copying becomes larger the more complex the system being imitated (the higher $K$ ). Therefore, imitation is not expected to be a successful strategy in markets in which firms compete on designs of high complexity, while in markets with designs of low complexity, imitation strategies are more successful, and thus more likely to be observed.

\footnotetext{
${ }^{5}$ Following the Central Limit Theorem (Kauffman, 1993: 53-54).

${ }^{6}$ This also holds for genetic algorithms that recombine of substrings of two parent strings to create a string (Holland, 1975; Birchenhall, 1995).

${ }^{7}$ Other search algorithms are greedy search (evaluating all $N$ neighbouring strings and choosing the one with highest fitness), extremal search (mutating the element with lowest fitness) and recombinant search (recombining of substrings of two parent strings to create a new string).
} 


\subsubsection{Constructional selection}

Fitness landscape models are most often used to understand the pros and cons of different search strategies in terms of the fitness of the optimum found (search benefits) and the time required to find an optimum (search costs). Typically, one assumes a given landscape with a particular architecture of epistatic relations (for example, using parameter $K$ ), which is kept constant through time. However, the structure of interdependencies of technological systems can also evolve over time, which has been called architectural innovation by Henderson and Clark (1990). Little research has been done on such endogenously changing architectures in fitness landscapes. One exception is a model of constructional selection developed by Altenberg $(1994 ; 1995)$. In a process of constructional selection fitness increases are realised not only through myopic search but also through a growth in the design space by means of the introduction of new elements in a system. This possibility should be regarded as an important and integral part of the long-run evolution of complex systems, because, in biological and artificial sciences alike, most systems grow in size. In the biological context, the growth of a design space is associated with genome growth. In technological evolution, design spaces also enlarge over time as the number of elements in technological artefact typically increases as the technology progresses (Clark, 1985).

The addition of a new element to an existing design has a very similar effect as a mutation in an extant element. Following the logic of fitness landscapes, the addition of a new element (with a particular allele, say 0 or 1 ), will generate new fitness values $w_{i}$ for those elements that are epistatically affected by the new element. The number of elements affected by a new element is called the pleiotropy of the new element. Altenberg $(1994 ; 1995)$ showed that the probability of increasing the total fitness of a string by adding a new element is inversely related to the pleiotropy of the new element. This can be understood from the disruptive effect of a new element with high pleiotropy. The higher the pleiotropy of an element, the more elements in the system will acquire a new fitness value by means of a random draw, and the more likely the mean of the newly drawn fitness values will be close to the expected value of 0.5 . As the fitness of the current string is always well above 0.5 , since it is a local optimum, the new total fitness value of the string with the new element will generally be lower than the old total fitness value. To increase the fitness of a string requires the new element to have a low pleiotropy, so that it becomes more likely to obtain a 'lucky' draw for new fitness values so that the new total fitness value to exceed the old total fitness value.

The NK-model of constructional selection has important theoretical implications with regard to the study of technological evolution (Frenken, 2005). It provides a theoretical understanding of the observation that technologies growing in size develop a core of highly interdependent elements developed early in time and a periphery with less interdependent elements added later in time (Clark, 1985). Selection favours elements that have a low degree of interdependency as to preserve the functioning of the core elements selected previously. For example, early car design evolved towards a dominant design with standardised core elements including particular variants of the engine, the transmission and steering. Safety belts, lights, airbags, air filters, catalytic converters, navigation systems are all elements added at a later stage of evolution. These elements greatly enhance the fitness of a car to its user environment in terms of safety, ease-of-use, environmental performance, etc. Yet, as explained by the model of constructional selection, and as emphasised by Clark (1985) and Saviotti (1996), the introduction of peripheral elements with low pleiotropy ('add-ons') does not require a fundamental adaptation of highpleiotropy 'core elements' that are fixed early on, the engine, the transmission, steering. Thus, Altenberg's model of constructional selection provides a new theoretical understanding of technological evolution that is compatible with earlier concepts of dominant design, which lacked a formal operationalisation hitherto. 


\subsection{Complex networks}

A second body of recent literature addresses the question how and to what extent network relations between innovating agents affect the rate of innovation and diffusion (for an extended survey, see Cowan, 2004). As with fitness landscapes models, one can distinguish between models of exogenous networks and models of endogenous formation of network structures.

More generally, the economics of networks has become an important topic of research as it relaxes some of the crude assumptions underlying mainstream economics. In standard economic theory, it is assumed that economic agents interact anonymously with all other agents through the market via prices. However, in many contexts, both economic and social, agents interact in networks where agents know each other's identity (Wasserman and Faust, 1994; Weisbuch et al., 2000). In the context of technological innovation, for example, studies have been done on coalitions, R\&D alliances and innovation networks (Axelrod et al., 1995; Duysters and Hagedoorn, 1996; Powell et al., 1996; Hagedoorn, 2002; Pyka and Kuppers, 2002; Breschi and Lissoni, 2003).

Apart from networks defined at the level of agents such as firms, the concept of networks is also applicable to technologies. In this context, one speaks of network technologies, in particular, information and transportation infrastructures (airports, railways, Internet, etc.). We will discuss a model of complex network technologies when discussing endogenous network formation.

\subsubsection{Small worlds}

Watts and Strogatz (1998) distinguish between three ideal-type of network structures: regular graphs where agents interact with their nearest neighbours, small worlds where most agents interact only with their nearest neighbours but some agents interact with agents that are not neighbours, and random graphs where agents interact with a random subset of other agents. Figure 2 shows all three network topologies, respectively. The three network topologies can be tuned by means of a single parameter $p$, which stands for the rewiring probability. Starting from a regular graph $(p=0)$, an increase in parameter $p$ means that edges will be rewired randomly with probability $p$. The small world is associated with a value of $p=0.1$. Increasing

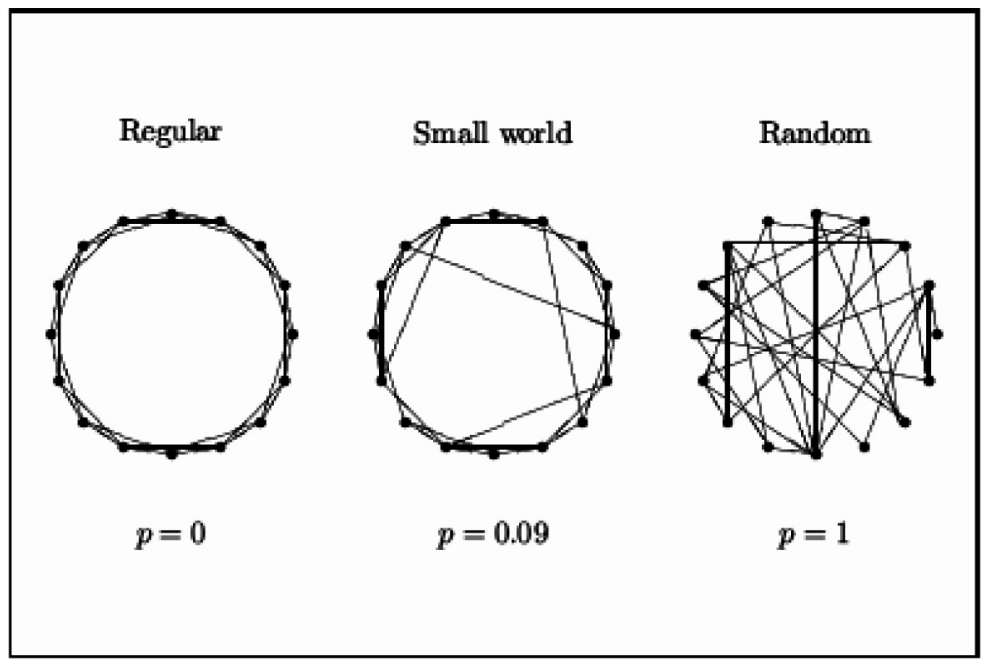

FIGURE 2 Network topologies as expressed by parameter $p$ (from: Cowan and Jonard, 2004). 
the parameter to the maximum value of $p=1.0$ leads us to the random network, because all edges get rewired in a random manner.

The properties of different network topologies can be analysed using two measures. The first is a measure of local density or 'cliquishness', which measures the extent to which an agent's neighbours are also neighbours (triangle relationships). Cliquishness is defined by:

$$
C=\frac{1}{N} \sum_{i} \sum_{\left(j, l \in \Gamma_{i}\right)} \frac{X(j, l)}{\left\|\Gamma_{i}\right\|\left(\left\|\Gamma_{i}\right\|-1\right) / 2}
$$

where $\Gamma_{i}$ is the set of neighbours of agent $i$ and $\left\|\Gamma_{i}\right\|$ is the size of the neighbourhood, and $X$ is either 0 (absent) or 1 (present). A second measure commonly used in average path length between any two agents:

$$
L=\frac{1}{N} \sum_{i} \sum_{j \neq 1} \frac{d(i, j)}{N-1}
$$

where $d(i, j)$ is the shortest path between $i$ and $j$ (i.e. the average number of steps separating two randomly chosen agents). Figure 3 shows the cliquishness and average path length for networks with different values of parameter $p$. Regular graphs $(p=0)$ have a high degree of cliquishness and a very long path length, whereas random graphs $(p=1)$ have a small degree of cliquishness and a very short path length. Interestingly, small worlds $(p=0.09)$ combine the two features, which explains why in small world networks (as the world social network is one) most people live in cliques yet information can still travel fast among these cliques as average path lengths are short. The combination of the two properties is made possible by the few agents who cut across cliques and hereby drastically shorten the average path length between any two agents in the network.

Even though many real networks have empirical small world properties of high cliquishness and short path length (Watts, 2003), the topic of small worlds has received relatively little

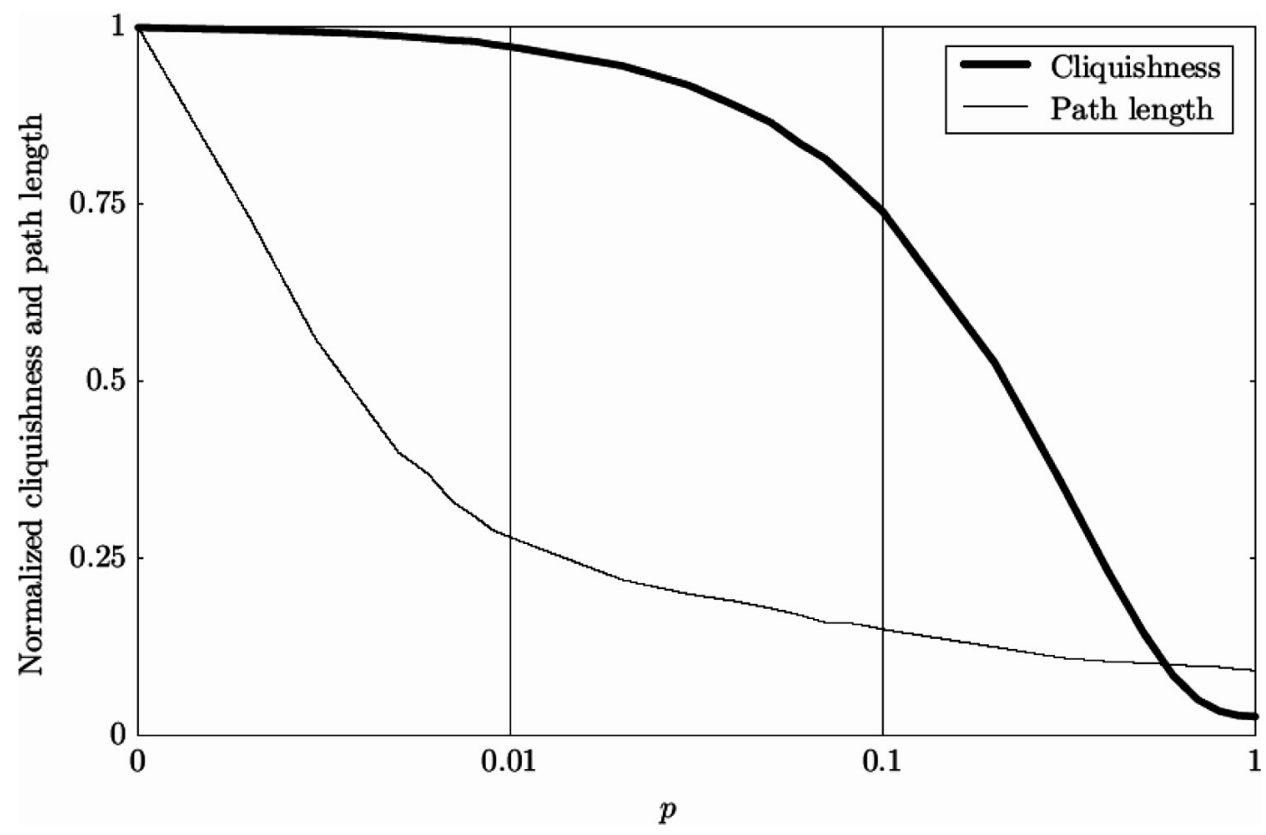

FIGURE 3 Properties of networks with different $p$-value (from Cowan and Jonard, 2004). 
attention of innovation economists so far. In the context of innovation, empirical studies on small world properties have been in co-authorship networks (Putsch, 2003) and on strategic technology alliances (Verspagen and Duysters, 2004). Theoretical contributions have come mainly from Cowan and Jonard $(2003 ; 2004)$ who developed models of knowledge creation in networks. The main contribution of their work is to show that small world networks are, generally, very supportive of knowledge production because of the combination of cliquishness and short path lengths. Cliques are supportive for knowledge creation as specialised knowledge production requires agents to form a community of practice sharing a common knowledge base. This is evident from the tightly connected networks of researchers (in collaboration networks) and tightly connected journals (in citation networks). However, too much cliquishness as in regular networks would hamper the diffusion of knowledge among specialised communities. Thus, the few agents connecting different cliques in small worlds make sure that interdisciplinary knowledge exchange can take place relatively easily. Cowan and Jonard $(2003 ; 2004)$ have elaborated on this idea in a series of models, which mainly differ in the nature of knowledge exchange (distinguishing between barter and broadcasting).

\subsubsection{Endogenous networks}

The model of small worlds, though empirically important, still lacks a proper theoretical foundation. ${ }^{8}$ One can speak of networks having the small world properties of high cliquishness and short path length, yet the question remains how these network structures emerge in the first place. In economics, most models of network formation follow a game-theoretical approach building on a seminal work by Jackson and Wolinsky (1996), who developed game-theoretical equilibrium concepts for agents interacting in networks. These papers are beyond the scope of the present paper, as these contributions are not primarily interested in technological innovation.

Another class of models of network formation follows an already seminal paper by Barabási and Albert (1999). The important difference between the Barabási-Albert model with the game-theoretical literature holds that not only the structure of interactions in the network evolves over time, but also the number of nodes participating in the network increases over time. Nodes enter sequentially to the network and have to decide to which of the nodes, which are already part of a network, to link. If their decision would be random, one would end up with a graph in which the degree of a node is proportional to time of entry of a node. Instead, Barabási and Albert (1999) proposed a different mechanism called preferential attachment to capture the bias of new nodes to connect with nodes with a high degree. The bias of new nodes to link to highly connected nodes follows from the benefits of short path length: establishing a connection to a node with many more connections means that many nodes can be reached quickly within two steps only.

The mechanism of preferential attachment implies that the probability of a new node to become connected to a node already present is proportional to the degree of latter node. Highly connected nodes thus tend to become even better connected over time. ${ }^{9}$ As a result, the network structure will evolve towards a very skewed distribution with few nodes capturing most links and most nodes having few links. Mathematically, these distributions follow a powerlaw, which implies that small occurrences are extremely common, whereas large instances are extremely rare. These distributions are scale free, which means that the network lacks a 'characteristic scale' so that, like fractals, smaller bits resemble the structure of the whole.

\footnotetext{
${ }^{8}$ In fact, it is more accurate to speak of small worlds properties than of small world models.

${ }^{9}$ This mechanism introduces a self-reinforcing rich-get-richer dynamics similar to the Arthur-model of increasing returns (Arthur, 1989).
} 
Albert and Barabási (2002) summarise empirical evidence on many networks exhibiting these features ranging from business networks, hyper-links on the web, and citation networks between scientists. The empirical evidence on scale free networks with a highly skewed distribution of links among nodes seems to be at odds with the empirical evidence on small worlds where nodes have similar degree. However, it is relatively simple to explain how small worlds can emerge in networks with preferential attachment by means of introducing geography, which often plays a role in networks because the costs of creating and maintaining a link increase with geographical distance. Human agents, for example, find it easier to maintain friendships when both agents live nearby (e.g. in a town), or see each other face-to-face on a regular basis (e.g. at work). Thus, geography counteracts preferential attachment in that it will cause the degree distribution to be less skewed.

Recent geographical extensions of the Barabási-Albert model proposed to model the probability of a new node linking to an existing node as being dependent both on the latter's degree and inversely on the geographical distance between the two nodes. Provided that the costs of distance are substantial, it means that the geography effect takes over the effect of preferential attachment. Though early entrants will make connections over long distance because nearby agents are still non-existent, nodes entering later in the network will typically connect with the nearest neighbours. In this way, the small world topology can be reproduced by the combination of the concept of preferential attachment and geography (Ozik et al., 2004).

Applications of the Barabási-Albert model to the topic of technological innovation lie primarily in the domain of network technologies. Most research has concentrated on explaining the growth dynamics of the Internet in terms of evolving complex network models. For example, the structure of hyperlinks between websites can be studied as an evolving network (Albert and Barabási, 2002). The physical infrastructure of network technologies can also be studied as an evolving network even though the time-scales are much longer than that of websites and links. In this context, the role of geography becomes an important parameter to explain the distribution of links. For example, a recent study (Guimera and Amaral, 2004) on the evolution of the airport network showed the applicability and extendibility of the Barabási-Albert framework by modelling the airport network as an evolving network with costs of distance thus reproducing a small worlds topology.

\subsection{Percolation}

Percolation models from physics are useful to model the dynamics of adoption (Stauffer and Aharony, 1994; Grebel, 2004) and of the role of spillovers in innovation (Silverberg and Verspagen, 2005). Although our focus here is on models of technological innovation, we will first discuss the basic percolation model as a model of technology adoption.

\subsubsection{Phase transitions}

Assume a simple case in which all agents have four nearest neighbours and that the neighbourhood relations are mutual. In terms of a graph, we assume a two-dimensional regular graph (lattice). Further assume that agents have different preferences, uniformly distributed between from 0 to 1 , for the product in question. The behavioural rules are specified as follows: if an agent gets informed about the existence of the new product, it evaluates whether the product's quality $q$ is equal or above its preference. If so, it adopts and informs its four nearest neighbours about the existence and quality of the product. If not, it does not adopt the product and does not inform its four nearest neighbours.

One can easily understand that the share of agents that will adopt a new product of quality $q$ (a value between 0 and 1), is at most $q$ because agents with a preference higher than $q$ will 
never adopt. However, since the information about the product needs to percolate through the network, there are conditions in which the actual share of agents adopting the product is lower than $q$, because some agents may prefer the product, but are never informed about it. It turns out that the critical value of $q$ above which the share of agents adopting the technology equals $q$, is $q^{*} 0.593$ (for a lattice). Below this value, many agents, who would have adopted the product provided that they knew about the product's existence, do not adopt because they are not informed about the product's existence.

Solomon et al. (2000) developed an interesting extension of this model. They assume that producers of a technology that is widely adopted $(q>0.593)$ have an incentive to lower the quality (to save on costs). They also assume that consumers having consumed a product with a certain quality will become more demanding in the future. A typical example of such dynamic are movies where producers exploit successful movies by making cheaper sequels, whereas consumers get more demanding as they learn to appreciate good movies. The authors show that under certain conditions, the market automatically evolves to products and preferences close to the critical threshold of 0.593 (a case of self-organisation). This model thus explains why many cultural products are just below or above the critical threshold, and, thus, are almost equally likely to become hits as to become flops.

A different application of percolation theory in the realm of technological innovation is the model by Silverberg and Verspagen (2005). They start from a lattice, which is bounded in the horizontal dimension, but unbounded in the vertical dimension. The horizontal dimension stands for technology neighbourhood (in principle, one could replace this simple neighbourhood structure with a Hamming space as in NK-models). The horizontal dimension is also anchored on a baseline with all technologies having zero performance (pre-technological age). The vertical dimension stands for technological performance. Using this two-dimensional space, one can model the dynamics of technological development using percolation principles. At each moment in time, each lattice site is in one out of four possible states: 0 (technologically impossible), 1 (possible but not yet discovered), 2 (discovered but not yet viable) and 3 (discovered and viable). The share of sites with value 0 , indicating impossible technologies, can also be introduced as a parameter to tune the difficulty of search. Note here that the presence of sites with value 0 comes close to the idea of valleys of low fitness in fitness landscape models.

The central idea in the Silverberg-Verspagen model holds that a site turns from 2 into 3 if and only if there exists a contiguous path of cells in state 2 or 3 connecting it to the baseline. Thus, a technology can become operational 'when it can draw on an unbroken chain of supporting technologies already in use'. Using the set-up, the dynamics of technological innovation can now be modelled as a local search process on the two-dimensional lattice. Sites that are 0 will always remain 0 , whereas sites that are 1 become 2 when they are discovered by local search and become 3 when there exists a contiguous path of cells in state 2 or 3 connecting it to the baseline. Because of the percolation properties of the model, local search will occasionally produce avalanches of many sudden innovations, as certain sites cause chain reactions of other sites becoming viable. In addition, because of the neighbour topology between technologies, such an avalanche will typically involve related technologies or a 'cluster' of technologies. These two properties, innovation avalanches in time and in clustering in technology space, reproduce two stylised facts from long wave research (Silverberg, 2003). In addition, the model provides an explanation for skewed distributions found in the citation impact of patents with only few patents getting almost all citations and most patents being cited only seldom (Jaffe and Trajtenberg, 2002). The highly cited patents would correspond to the sites that set in motion an avalanche of innovation in the neighbourhood. 


\subsubsection{Self-organised criticality}

As it is the case for NK landscapes and network models, one can again distinguish between percolation models with exogenous complexity and endogenous complexity. A model by Plouraboue et al. (1998) can be regarded as an example of the use of endogenous complexity in the context of technology adoption. They developed a model of technology adoption where an agent's neighbours affect the decisions of each agent. The endogenous component in the model concerns the weights that an agent assigns to the decision of each of its neighbours. These weights change over time according to past influence: a neighbour that successfully influenced an individual to adopt a particular technology in the past, becomes a more important neighbour in the future in terms of the weight assigned to this neighbour (as in neural network models). The type of reinforcement learning is evolutionary in nature in that decisions are based according the past experiences rather than strategic interaction, and decisions are myopic in nature in that agents lack foresights of future payoffs. ${ }^{10}$

The important contribution of their model has been to show that a small number of agents adopting the new technology will not lead to percolation of the technology throughout the population. As the network evolves in terms of the weights each agent assigns to each neighbour, a structure emerges spontaneously in which some agents heavily influence the decision of its neighbours, which also influence their neighbours, etc. As a result, giving the new technology to these 'high-influence' agents can then set in motion an avalanche of adoption decisions, which lead to the percolation of the new technology throughout the population. The endogenous formation of a network that is capable of efficiently distributing information is an example of self-organised criticality in which the phase transition is not pre-determined by a parameter setting, as in the standard percolation case, but emerges from the dynamics of the network itself. Although this model deals with adoption and diffusion, rather than with technological innovation, the principle underlying self-reinforcing criticality and avalanches can also be applied to the domain, for example, in the context of technological paradigm shifts.

\section{PROSPECTS AND CHALLENGE}

Complexity theory can be regarded as an emerging paradigm for understanding the complex dynamics underlying processes of technological innovation. ${ }^{11}$ Technology is a complex system par excellence: technologies are complex systems made out of many interacting parts. In addition, technological innovation is more often than not a collective process in which agents are engaged in a process of mutual learning. The precise topology of the network interactions between agents thus bears important implications for the efficiency and effectiveness of collective learning processes.

Complexity models have the advantage of capturing more realistic features of the innovation process, while avoiding the danger of over-parameterisation. Typically, complexity models start from a specification with only one critical parameter (the $K$-parameter in NK-models,

\footnotetext{
${ }^{10}$ Also known as neural networks (Plouraboue et al., 1998).

${ }^{11}$ There have been important recent advances in the establishment of complexity science in the social sciences as a field of scientific investigation. A number of complexity-minded journals started recently including free electronic journals like JASSS (http://jasss.soc.surrey.ac.uk/JASSS.html) and e-JEMED (http://beagle.u-bordeaux4.fr/jemed/). Web portals are available on agent-based modelling (http://www.econ.iastate.edu/tesfatsi/atechevo.htm) and networks (http://www.econ.iastate.edu/tesfatsi/anetwork.htm). A simulation platform is available from SWARM (http://www.swarm.org/). More specifically tailored to the community of evolutionary economics is a more recent initiative called the Laboratory for Simulation Development (http://www.business.auc.dk/lsd/). See also Valente and Andersen (2002).
} 
the $q$-parameter in percolation models or the $p$-parameter in network models). As analytical solutions are more often than not impossible to obtain due to non-linearity and stochasticity, a small number of parameters still allow one to understand a model's behaviour well by simulation. Complexity modelling should thus not be mistaken for complicated models with many parameters and possible behaviours (Axelrod, 1997).

\subsection{Research avenues}

Applications of complexity theory to the topic of technological innovation lie in three 'core' approaches: fitness landscape models, complex network models and percolation models. Given these core approaches, research avenues can be developed in at least four directions:

1. Applying core models on topics other than yet related to technological innovation. Promising new topics include economic geography (Arthur, 1990; Andersson et al., 2003; 2005), evolutionary economics of demand (Cowan et al., 1997; Aversi et al., 1999), the evolutionary theory of the firm (Levinthal, 1997; Marengo et al., 2000), econometrics (Foster and Wild, 1999; Foster, 2004), evolutionary macroeconomics (Llerena and Lorentz, 2004) and environmental economics (van den Bergh, 2004).

2. Recombining the three core approaches. An example is a model in which agents search a fitness landscape in a decentralised way with different agents responsible for designing different parts of a technological system (Kauffman and Macready, 1995; Rivkin and Siggelkow, 2003; Frenken and Valente, 2004) or a model in which agents form networks while searching on complex fitness landscapes (Fagiolo et al., 2005). A possibility yet to be explored is a model that addresses percolation in complex rather than nearest-neighbour networks.

3. Recombining core models from complexity sciences with earlier evolutionary models of industry dynamics (Nelson and Winter, 1982; Klepper, 1996). For example, one could use a standard Nelson-and-Winter set-up and introduce an NK fitness landscape instead of the traditional smooth search space in capital-labour space.

4. Empirical testing of core models. Confronting complexity models with empirical data, however, can be done in more than one way. In some applications, hypotheses can be derived deductively as outcomes of the model. For example, Fleming and Sorenson (2001) specified hypotheses based on the NK-model regarding the success rate of patents with varying degrees of complexity. In most instances, however, empirical validation of complexity models follows a different methodology based on a model's capacity to replicate stylised facts. Some of the methodological issues related to empirical verification of complexity models in more detail are discussed subsequently.

\subsection{Empirical verification}

A topic that deserves attention is the interplay between simulation modelling and empirical research (Pyka and Fagiolo, 2005; Valente, 2005). There are many possible interfaces between the two types of research ranging from calibration, to econometric hypothesis testing, to the reproduction of stylised facts (typically done in evolutionary economics), to the improvement of the empirical foundations for agents' behaviour. Note that calibration also comes in at least two forms: one can calibrate a model with initial conditions or one can calibrate parameters. Also note that simulation models can be used for policy design as well: one can create an artificial world in which policy experiments can take place to evaluate, for a large range of behavioural rules, the effectiveness and robustness of new policy (e.g., anti-trust policies, environmental policies, traffic rules, etc.). 
In complexity research scholars, most use empirical data in the form of 'stylised facts', which are rather well-established empirical regularities found in many different industries, countries, or periods in time and which require a theoretical explanation. Some examples are skewed firm-size distributions, clustering of innovative activity in time and space, the concentration of firms in particular regions etc. Complexity models are important tools to understand the processes that give rise to such regularities. However, stylised facts are often over identified: 'the facts can be generated by a wide range of distinct models' (Pumain, 2004, p. 1). Scaling laws, for example, can be reproduced by many different models with more or less equal accuracy. In such cases, one needs additional criteria to select among possible candidate models. 'It is essential to select specifications that integrate in the model a significant part of the existing knowledge' about the topic in question (Pumain, 2004, p. 1). A complementary strategy is to build models with the intention to replicate multiple stylised facts in a single model for a wide range or probable range of parameter values (e.g. Aversi et al., 1999; Fagiolo et al., 2004). A 'good' model in this view in a model that performs well in the replication of the largest number of stylised facts. In addition, when a new stylised fact becomes established, one can then evaluate to what extent existing models are able to account for this new stylised fact to evaluate the 'robustness' of models vis-à-vis new facts. We can head all these criteria under the label of replication-of-stylised-facts (ROSF) criterion.

A second criterion often used by evolutionary economists to distinguish a 'good' model from a 'bad' one is the realism of the assumptions (ROAS) that underlie the model (Mäki, 1994). In this view, modelling assumptions, such as behavioural ones, should not contradict widely accepted empirical evidence about agents' behaviour. In this respect, simulation models differ from neoclassical economics, in which the plausibility of assumptions is less important. Most neoclassical economists adhere, implicitly or explicitly, an instrumentalist philosophy of science advocated by Friedman (1953), according to which the prime quality criterion of models is to predict accurately.

A third methodological quality criterion can be labelled KISS. The KISS principle stands for 'Keep it Simple, Stupid'. This principle is primarily a modelling recommendation to start from a simple model that can be well understood by simulation and/or through analytical solutions, and only then develop the model in more specific directions. ${ }^{12}$ The KISS principle can also be judged ex post to see whether a model adhered to the principle by asking oneself whether this model is really the simplest model possible that is capable of producing the desired outcomes (and given the criteria of ROSF and ROAS)? One may argue that, in principle, simpler models with few parameters, are better amenable to be confronted with empirical data than more complicated models, even though the latter are often motivated by the wish to capture more aspects of reality.

A fourth criterion can be called TAPAS which stands for 'Take A Previous model and Add Something'. Put differently, incremental strategies in modelling are often most successful. As for the KISS principle, the TAPAS principle is primarily meant as a modelling heuristic, yet it can also be regarded as an ex post evaluation criterion. One would prefer a modelling approach that builds on existing models and assumptions as to maximise diffusion and cumulativeness of knowledge. Too often, models start from scratch with many 'idiosyncratic' elements, which take a lot of time to construct and which are hard to communicate. In contrast, models that extend existing 'core models' are faster to build and more easily understood by other people. This should, of course, not be taken to mean that experimentation with new models is to be

12 The KISS principle has recently been challenged by the KIDS principle (Edmonds and Moss 2004), referring to 'Keep it Descriptive Stupid'. The KIDS approach simulates phenomena in the most straightforward way possible, taking into account the widest possible range of evidence. Simplification is only applied when and if the model and evidence justify this. In contrast, the Kiss principle advocates that one should start with the simplest possible model and later extended in necessary. 
discouraged, but rather that one can take advantage of existing core models to formulate a new robust model in a relatively short amount of time and with a larger degree of understanding by others. The principles of KISS and TAPAS combined indeed suggest that an important part of modelling should be devoted to building core KISS-type models that can be easily extended along the TAPAS principle.

\section{Acknowledgements}

This paper has been written during the EXYSTENCE Thematic Institute on 'Innovation and Complexity', Tech Gate, Vienna, 6 September-8 October 2004 funded by the European Commission under no. IST-2001-32802. I thank Giorgio Fagiolo, Cristiano Antonelli and three anonymous referees for their helpful comments. The usual caveats apply.

\section{References}

Albert, R. and Barabási A.L., (2002) Statistical mechanics of complex networks. Reviews of Modern Physics, 74, 67-97.

Altenberg, L. (1994) Evolving better representations through selective genome growth. In Schaffer, J.D., Schwefel, H.P. and Kitano H. (eds.) Proceedings of the IEEE World Congress on Computational Intelligence. Piscataway, NJ: IEEE, pp. 182-187.

Altenberg, L. (1995) Genome growth and the evolution of the genotype-phenotype map. In Banzhaf W. and Eckman F.H. (ed.) Evolution and Biocomputation. Berlin \& Heidelberg: Springer-Verlag, pp. 205-259.

Altenberg, L. (1997) NK fitness landscapes. In Back, T., Fogel, D., Michalewicz, Z., (eds.) The Handbook of Evolutionary Computation. Oxford: Oxford University Press.

Anderson, P.W., Arrow, K.J. and Pines, D. (eds.) (1988) The Economy as an Evolving Complex System. Reading, Mass.: Addison-Wesley.

Andersson, C., Hellervik, A., Lindgren, K., Hagson, A. and Tornberg, J. (2003) Urban economy as a scale-free network. Physical Review E, 68(3): 036124 Part 2.

Andersson, C., Frenken, K. and Hellervik, A. (2005) A complex network approach to urban growth. Papers in Evolutionary Economic Geography \#05.05, Available Online at: http://econ.geog.uu.nl/peeg/peeg.html.

Arthur, W.B. (1989) Competing technologies, increasing returns, and lock-in by historical events. Economic Journal, 99, 116-131.

Arthur, W.B. (1990) Silicon Valley locational clusters: when do increasing returns imply monopoly? Mathematical Social Sciences, 19(3), 235-251.

Arthur, W.B., Durlauf, S.N. and Lane, D.A. (eds.) (1997) The Economy as an Evolving Complex System II. Reading, Mass.: Addison-Wesley.

Atkinson, A.B. and Stiglitz, J.E. (1969) A new view of technological change. Economic Journal, 79, 573-578.

Aversi, R., Dosi, G., Fagiolo, G., Meacci, M. and Olivetti, C. (1999) Demand dynamics with socially evolving preferences. Industrial and Corporate Change, 8(2), 353-408.

Auerswald, P., Kauffman, S., Lobo, J. and Shell, K. (2000) The production recipes approach to modeling technological innovation: an application to learning-by-doing. Journal of Economic Dynamics and Control, 24, 389-450.

Axelrod, R. (1997) The Complexity of Cooperation. Princeton: Princeton University Press.

Axelrod, R., Mitchell, W., Thomas, R.E., Bennett, D.S. and Bruderer, E. (1995) Coalition formation in standard-setting alliances. Management Science, 41(9), 1493-1508.

Barabási, A.L. and Albert, R. (1999) Emergence of scaling in random networks. Science, 286, 509-512.

Bikhchandani, S., Hirshleifer, D. and Welch, I. (1998) Learning from the behavior of others: Conformity, fads, and informational cascades. Journal of Economic Perspectives, 12(3), 151-170.

Birchenhall, C. (1995) Modular technical change and genetic algorithms. Computational Economics, 8, $233-253$.

Breschi, S. and Lissoni, F. (2003) Mobility and social networks: localised knowledge spillovers revisited. CESPRI Working Paper 142, Available Online at: www.cespri.it.

Bruckner, E., Ebeling, W., Jiménez, Montãno, M.A. and Scharnhorst, A. (1996) Nonlinear stochastic effects of substitution - an evolutionary approach. Journal of Evolutionary Economics, 6, 1-30.

Clark, K.B. (1985) The interaction of design hierarchies and market concepts in technological evolution. Research Policy, 14, 235-251.

Cowan, R. (2004) Network models of innovation and knowledge diffusion, MERIT Research Memorandum RM2004016, Available Online at: www.merit.unimaas.nl.

Cowan, R., Cowan, W. and Swann, P. (1997) A model of demand with interaction between consumers. International Journal of Industrial Organisation, 15, 711-732.

Cowan, R. and Jonard, N. (2003) The dynamics of collective invention. Journal of Economic Behaviour and Organization, 52(4), 513-532. 
Cowan, R. and Jonard, N. (2004) Network structure and the diffusion of knowledge. Journal of Economic Dynamics and Control, 28(8), 1557-1575.

Dalle, J.-M. (1997) Heterogeneity vs. externalities in technological competition: a tale of possible technological landscapes. Journal of Evolutionary Economics, 7, 395-413.

David, P.A. (1975) Technical Choice, Innovation and Economic Growth. Essays on American and British Experience in the Nineteenth Century. London: Cambridge University Press.

David, P.A. (1993) Path-dependence and predictability in dynamic systems with local network externalities: a paradigm for historical economics. In Foray, D. and Freeman, C. (eds.), Technology and the Wealth of Nations. London \& New York: Pinter, pp. 208-231.

Dawid, H. (2004) Agent-based models of innovation and technological change. In Tesfatsion, L. and Judd, K. (eds.) Handbook of Computational Economics II: Agent-based Computational Economics, in press.

Dosi, G., Levinthal, D. and Marengo, L. (2003) Bridging contested terrain: linking incentive-based and learning perspectives on organizational evolution. Industrial and Corporate Change, 12(2), 413-436.

Duysters, G. and Hagedoorn, J. (1996) Internationalization of corporate technology through strategic partnering: An empirical investigation. Research Policy, 25(1), 1-12.

Edmonds, B. and Moss, S. (2004) From KISS to KIDS - an 'anti-simplistic' modelling approach. CPM Report no. 04-132, 1 April, Available Online at: http://cfpm.org/cpmreps.html.

Fagiolo, G. and Dosi, G. (2003) Exploitation, exploration and innovation in a model of endogenous growth with locally interacting agents. Structural Change and Economic Dynamics, 14(3), 237-273.

Fagiolo, G., Dosi, G. and Gabriele, R. (2004) Matching, bargaining, and wage setting in an evolutionary model of labor market and output dynamics. Advances in Complex Systems, 7(2), 157-186.

Fagiolo, G., Marengo, L. and Valente, M. (2005) Population learning in a model with random payoff landscapes and endogenous networks. Computational Economics, in press.

Fisher, J.C. and Pry, R.H. (1971) A simple substitution model of technical change. Technological Forecasting and Social Change, 3, 75-81.

Fleming, L. and Sorenson, O. (2001) Technology as a complex adaptive system: evidence from patent data. Research Policy, 30, 1019-1039.

Foster, J. (2004) Econometrics and evolutionary economics. In Foster, J. and Hölzl, W. (eds.), Applied Evolutionary Economics and Complex Systems. Cheltenham: Edward Elgar, pp. 15-35.

Foster, J. and Hölzl, W. (eds.) (2004), Applied Evolutionary Economics and Complex Systems. Cheltenham: Edward Elgar.

Foster, J. and Wild, P. (1999) Econometric modelling in the presence of evolutionary change. Cambridge Journal of Economics, 23(6), 749-770.

Frenken, K. (2005) Innovation, Evolution and Complexity Theory. Cheltenham \& Northampton, Mass: Edward Elgar, in press.

Frenken, K., Marengo, L. and Valente, M. (1999) Interdependencies, near-decomposability and adaptation. In: Brenner, T. (ed.) Computational Techniques for Modelling Learning in Economics. Boston: Kluwer Academic Publishers, pp. 145-165.

Frenken, K. and Nuvolari, A. (2004) The early history of steam engine technology: an evolutionary interpretation using complexity theory. Industrial and Corporate Change, 13(2), 419-450.

Frenken, K. and Valente, M. (2004) A fitness landscape approach to decentralised problem-solving, mimeo, Utrecht University, 12 February.

Friedman, M. (1953) Methodology of positive economics. Essays in Positive Economics. Chicago: University of Chicago Press, pp. 3-43.

Gavetti, G. and Levinthal, D. (2000) Looking forward and looking backward: cognitive and experiential search. Administrative Science Quarterly, 45(1), 113-137.

Grebel, T. (2004) Entrepreneurship: A New Perspective. London \& New York: Routledge.

Guimera, R. and Amaral, L.A.N. (2004) Modeling the world-wide airport network. European Physical Journal B, 38(2), 381-385.

Hagedoorn, J. (2002) Inter-firm R\&D partnerships: an overview of major trends and patterns since 1960. Research Policy, 31(4), 477-492.

Hartmann-Sonntag, I., Scharnhorst, A. and Ebeling, W. (2004) Modelling self-organization and innovation processes in networks, ArXiv: cond-mat/0406425 v1, 18 June.

Henderson, R. and Clark, K. (1990) Architectural innovation. Administrative Science Quarterly, 35, 9-30.

Holland, J.H. (1975) Adaptation in Natural and Artificial Systems. Ann Arbor: University of Michigan Press.

Hovhannisian, K. (2004) Imperfect local search strategies on technology landscapes: satisfying, deliberate experimentation and memory dependence. ROCK Working Paper, University of Trento.

Hovhannisian, K. and Valente, M. (2004) Modeling directed local search strategies on technology landscapes: depth and breadth. ROCK Working Paper, University of Trento.

Jackson, M.O. and Wolinsky, A. (1996) A strategic model of social and economic networks. Journal of Economic Theory, 71, 44-74.

Jaffe, A. and Trajtenberg, M. (2002) Patents, Citations \& Innovations. A Window on the Knowledge Economy. Cambridge, Mass: MIT Press.

Kauffman, S.A. (1993) The Origins of Order. Self-Organization and Selection in Evolution. New York \& Oxford: Oxford University Press. 
Kauffman, S.A. and Macready, W.G. (1995) Technological evolution and adaptive organizations. Complexity, 1, 26-43.

Kauffman, S.A., Lobo, J. and Macready, W.G. (2000) Optimal search on a technology landscape. Journal of Economic Behavior and Organization, 43, 141-166.

Klepper, S. (1996) Entry, exit, growth and innovation in the product life cycle. American Economic Review, 86, $562-583$

Kwasnicki, W. (1996) Knowledge, Innovation, and Economy. An Evolutionary Exploration. Cheltenham: Edward Elgar.

Levinthal, D.A. (1997) Adaptation on rugged landscapes. Management Science, 43, 934-950.

Levinthal, D.A. and Warglien, M. (1999) Landscape design: designing for local action in complex worlds. Organization Science, 10, 342-357.

Leydesdorff, L. and van den Besselaar, P. (eds) (1994) Evolutionary Economics and Chaos Theory: New Directions in Technology Studies. London: Pinter.

Llerena, P. and Lorentz, A. (2004) Alternative theories on economic growth and the co-evolution of macro-dynamics and technological change: A survey, LEM Working Paper 2003/28, Pisa.

Mäki, U. (1994) Reorienting the assumptions issue. In Backhouse, R. (ed.) New Directions in Economic Methodology. London and New York: Routledge, pp. 236-256.

Malerba, F., Nelson, R.R., Orsenigo, L. and Winter, S.G. (1999) History friendly models of industry evolution: the case of the computer industry. Industrial and Corporate Change, 8, 3-40.

Marengo, L., Dosi, G., Legrenzi, P. and Pasquali, C. (2000) The structure of problem-solving knowledge and the structure of organizations. Industrial and Corporate Change, 9, 757-788.

McCarthy, I.P. (2003) Technology management - a complex adaptive systems approach, International Journal of Technology Management, 25(8), 728-745.

McCarthy, I.P. (2004) Manufacturing strategy: understanding the fitness landscape. International Journal of Operations \& Production Management, 24(1-2), 124-150.

Metcalfe, J.S. (1988) The diffusion of innovations: an interpretative survey. In Dosi, G., Freeman, C., Nelson, R., Silverberg, G. and Soete, L. (eds.) Technical Change and Economic Theory. London: Pinter, pp. 560-589.

Nelson, R.R. and Winter, S.G. (1982) An Evolutionary Theory of Economic Change. Cambridge, Mass. \& London: Belknap Press of Harvard University Press.

Ozik, J., Hunt, B.R. and Ott, E. (2004) Growing networks with geographical attachment preference: Emergence of small worlds. Physical Review E, 69(2): Art. No. 026108 Part 2, February.

Page, S.E. (1996) Two measures of difficulty. Economic Theory, 8, 321-346.

Plouraboue, F., Steyer, A. and Zimmerman, J.-B. (1998) Learning induced criticality in consumers' adoption pattern: a neural network approach. Economics of Innovation and New Technology, 6, 73-90.

Powell, W.W., Koput, K.W. and Smith-Doerr, L. (1996) Interorganizational collaboration and the locus of innovation: networks of learning in biotechnology. Administrative Science Quarterly, 41(1), 116-145.

Pumain, D. (2004) Scaling laws and urban Systems. Santa Fe Working Paper 04-02-002, Available Online at: www.santafe.edu.

Putsch, F. (2003) Analysis and modeling of science collaboration networks. Advances in Complex Systems, 6(4), 477-485.

Pyka, A. and Fagiolo, G. (2005) Agent-Based Modelling: A Methodology for Neo-Schumpeterian Economics, Discussion Paper Series 272, University of Augsburg, Department of Economics, Available Online at: www.wiwi.uni-augsburg.de/vwl/institut/inst-forsch/.

Pyka, A. and Kuppers, G. (eds.) (2002) Innovation Networks: Theory and Practice. Cheltenham: Edward Elgar.

Rivkin, J.W. (2000) Imitation of complex strategies. Management Science, 46, 824-844.

Rivkin, J.W. (2001) Reproducing knowledge: replication without imitation at moderate complexity. Organization Science, 12(3), 274-293.

Rivkin, J.W. and Siggelkow, N. (2003) Balancing search and stability: Interdependencies among elements of organizational design. Management Science, 49(3), 290-311.

Saviotti, P.P. (1996) Technological Evolution, Variety and the Economy Cheltenham \& Brookfield: Edward Elgar.

Silverberg, G. (2003) Long waves: conceptual, empirical and modelling issues, In Hanusch, H. and Pyka, A. (eds.) The Elgar Companion to Neo-Schumpeterian Economics. Cheltenham: Edward Elgar, (also MERIT Research Memorandum RM2003-015, in press, University of L'Aquila, Italy). Available Online at: www.merit.unimaas.nl.

Silverberg, G., Dosi, G. and Orsenigo, L. (1988) Innovation, diversity and diffusion: a self-organisation model. Economic Journal, 98, 1032-1054.

Silverberg, G. and Verspagen, B. (1994) Learning, innovation and economic growth: a long-run model of industrial dynamics. Industrial and Corporate Change, 3, 199-223.

Silverberg, G. and Verspagen, B. (2005) A percolation model of innovation in complex technology spaces. Journal of Economic Dynamics and Control, 29(1-2), 225-244.

Simon, H.A. (1969) The Sciences of the Artificial. Cambridge, Mass. \& London: MIT Press, 3rd edn. 1996.

Simon, H.A. (2002) Near decomposability and the speed of evolution. Industrial and Corporate Change, 11, 587-599.

Sommer, S.C. and Loch, C.H. (2004) Selectionism and learning in projects with complexity and unforeseeable uncertainty. Management Science, 50(10), 1334-1347.

Stauffer, D. and Aharony, A., (1994) Introduction to Percolation Theory. London: Taylor and Francis.

Solomon, S., Weisbuch, G., de Arcangelis, L., Jan, N. and Stauffer, D. (2000) Social percolation models. Physica A, 277(1-2), 239-247. 
Teece, D., Pisano, G. and Shuen, A. (1997) Dynamic capabilities and strategic management. Strategic Management Journal, 18(7), 509-533.

Valente, M. (2005) Qualitative simulation modelling. Mimeo, 9 February.

Valente, M. and Andersen, E.S. (2002) A hands-on approach to evolutionary simulation: Nelson and Winter models in the Laboratory for Simulation Development. The Electronic Journal of Evolutionary Modeling and Economic Dynamics, 1(15), 1003, Available Online at: http://beagle.u-bordeaux4.fr/jemed/.

van den Bergh, J. (2004) Evolutionary thinking in environmental economics: retrospect and prospect. In Foster, J. and Hölzl, W. (eds.), Applied Evolutionary Economics and Complex Systems. Cheltenham: Edward Elgar, pp. 239-275.

Verspagen, B. and Duysters, G. (2004) The small worlds of strategic technology alliances. Technovation, 24(7), 563-571.

Wasserman, S. and Faust, K. (1994) Social Network Analysis: Methods and Applications. Cambridge: Cambridge University Press.

Watts, D.J. (2003) Six Degrees: The Science of a Connected Age. New York, Norton.

Watts, D.J. and Strogatz, S. (1998) Collective dynamics of small-world networks, Nature, 393, 440-442.

Weisbuch, G., Kirman, A. and Herreiner, D. (2000) Market organisation and trading relationships. The Economic Journal, 110, 411-436.

Windrum, P. (2004) Neo-Schumpeterian simulation models. In Hanusch, H. and Pyka, A. (eds.), The Elgar Companion to Neo-Schumpeterian Economics. Cheltenham: Edward Elgar, (also MERIT Research Memorandum RM2004002, in press). Available Online at: www.merit.unimaas.nl.

Winter, S.G. (1984) Schumpeterian competition in alternative technological regimes. Journal of Economic Behavior and Organization, 5, 287-320.

Wright, S. (1932) The roles of mutation, inbreeding, crossbreeding, and selection in evolution. Proceedings of the Sixth International Congress on Genetics, 1, 356-366. 